\title{
Lassen sich GKV-Routinedaten nutzen, um auf leitliniengerechte Versorgung zu schließen?
}

\author{
Eine Analyse am Beispiel der arteriellen Hypertonie
}

\section{SVEJA EBERHARD}

Dr. Sveja Eberhard ist Leiterin Politik und Versorgungsforschung bei der AOK Niedersachsen in Hannover

\author{
Seit der Einführung des § 75 im SGB X werden \\ Krankenkassen-Routinedaten zunehmend herangezogen, \\ um Fragen aus der Versorgungsforschung zu \\ beantworten. Häufig ist jedoch noch unklar, ob und in \\ welchem Umfang sich diese Daten eignen, um bestimmte \\ Fragestellungen zu beantworten. Im folgenden Beitrag \\ wird untersucht, inwiefern es möglich ist, mit Hilfe \\ von GKV-Routinedaten auf eine leitlinienadäquate \\ Versorgung von Patienten mit arterieller Hypertonie \\ zu schließen. Dazu werden die diagnostischen und \\ therapeutischen Aspekte einer Hypertonietherapie \\ den dazu verfügbaren GKV-Daten gegenübergestellt.
}

\section{Hintergrund}

Das Ziel von Leitlinien ist es, Hilfestellung bei Entscheidungen während des Behandlungsprozesses zu geben. Leitlinien geben dabei auf Basis wissenschaftlicher Erkenntnisse entwickelte Empfehlungen in Form von Handlungskorridoren wider, die rechtlich nicht bindend sind. Gemäß der AWMF (Arbeitsgemeinschaft der Wissenschaftlichen Medizinischen Fachgesellschaften) sollen sie zum einen für mehr Sicherheit in der Medizin sorgen, zum anderen aber auch ökonomische Aspekte berücksichtigen. Ob oder welche spezifischen Empfehlungen aus Leitlinien in der Praxis tatsächlich umgesetzt werden, ist jedoch häufig nicht bekannt (Frank \& Konta 2005). Eine erhöhte Transparenz über diese Frage wäre entscheidend, um Versorgungsprozesse verbessern zu können und Leitlinien praxisgerecht weiterzuentwickeln.
Primärerhebungen in Arztpraxen zu dieser Frage sind aufwändig, haben oft nur sehr geringe Rücklaufquoten und weisen methodische Probleme auf. GKV-Daten, die viele Informationen zu Behandlungsprozessen und zur Morbidität umfassen, haben demgegenüber den Vorteil, dass sie bereits vorliegen, dass die Forschungsfrage keinen Einfluss auf die Daten hat und durch ihre Vollständigkeit ein Selektionsoder Erinnerungsbias weitgehend entfällt. Somit stellt sich die Frage, ob man diese Daten nutzen kann, um Erkenntnisse über die Umsetzung von Leitlinien im Versorgungsalltag zu erhalten: Chancen aber auch Limitationen sollen in diesem Beitrag am Beispiel der arteriellen Hypertonie (Bluthochdruckerkrankung) verdeutlicht werden. Die Erkrankung wurde ausgewählt, da sie die häufigste Diagnose in Allgemeinarztpraxen und bei hausärztlich und fachärztlich tätigen Internisten widerspiegelt (ZI 2010) und seit Jahren 
verschiedene Leitlinien für die Behandlung der Hypertonie vorliegen. Zudem ist die Hypertonieversorgung laut Literatur von erheblicher Unter- und Fehlversorgung geprägt, so dass ein regelmäßiges Monitoring der Versorgung Qualitätspotenziale erschließen könnte (Wehling 2010, DHL 2009, RKI 2008, Wittchen et al. 2003).

Derzeit existieren in Deutschland zwei Leitlinien unterschiedlicher Herausgeber, zum einen die S-2 Leitlinie der Deutschen Gesellschaft für Hypertonie und Prävention - Deutsche Hochdruckliga e.V. (DHL) von 2008 mit Addendum vom Dezember 2011, sowie die Therapieempfehlungen der Arzneimittelkommission der deutschen Ärzteschaft (Akd Ä) von 2004. Zudem wurde 2010 mit der Arbeit an der Nationalen Versorgungsleitlinie Hypertonie begonnen (BÄK et al. 2012 unter http://www.versorgungsleitlinien. de/themen/hypertonie/).

\section{Eine strikte Umsetzung aller Empfehlungen könnte ins Leere laufen und nicht den gewünschten Qualitätszuwachs bringen.}

adäquaten Beurteilung zu kommen. Ferner folgt daraus, dass eine Untersuchung der Versorgungslage nicht nur fragen sollte, welcher Anteil der Patienten nicht leitliniengerecht behandelt wird, sondern auch, welche Patienten keine leitliniengerechte Therapie erhalten bzw. ob und mit welchen Merkmalen sie sich von den anderen Patienten unterscheiden. Eine von den Leitlinien abweichende Therapie kann sowohl in den Wünschen und Merkmalen des Patienten begründet sein, als auch die Präferenzen der Behandelnden widerspiegeln. Untersuchungen aus den USA zeigen, dass die Versorgung von Patienten bei gleicher Diagnose je nach sozioökonomischem Status, Geschlecht, Wohnort und Behandlungszentrum sehr unterschiedlich ausfällt (Murphy et al. 2009). Regionale Transparenz in Hinsicht auf die Umsetzung von Leitlinienempfehlungen könnte somit Hinweise darauf geben, wo und ob sich die Versorgung ggf. verbessern ließe.

\section{Nutzung von GKV- Routinedaten für Forschungszwecke}

Derzeit sind rund 85 Prozent der Bevölkerung in Deutschland in einer gesetzlichen Krankenkasse versichert (KM6-Statistik, Stand 1. Juli 2012). Krankenkassen

Vorab zu berücksichtigen ist, dass gerade die Versorgung der Hypertonie durch eine hohe Komplexität gekennzeichnet ist, da die meisten hypertensiven Patienten multimorbid sind und die gleichzeitige Einhaltung von mehreren, jeweils auf einzelne Krankheiten fokussierende Leitlinien unter Umständen für die Patienten kontraproduktiv sein könnte (Fitzgerald \& Bean 2010). Eine Forderung nach strikter Umsetzung aller Empfehlungen würde in diesem Fall ins Leere laufen und eben nicht den erwünschten Qualitätszuwachs bringen. Dennoch können die Empfehlungen in Leitlinien als Maßstab zur Orientierung dienen - Abweichungen von diesem Maßstab können dann statistisch beurteilt werden, wenn sie auffällig häufig oder besonders selten vorliegen (Abholz 2006). Für eine Beurteilung der Prozessqualität sind die dargestellten Einschränkungen somit kein grundsätzliches Hindernis, sie müssen aber bei Operationalisierungen von Beurteilungsmaßstäben berücksichtigt werden. So ließe sich beispielsweise ein Bereich der Toleranz um einen Indikator legen, um zu einer speichern für die Erfüllung ihres gesetzlichen Auftrages eine Vielzahl von Abrechnungsdaten, welche auch Informationen umfassen, die wichtige Erkenntnisse zum Gesundheitszustand der Bevölkerung bzw. zum Leistungsgeschehen und damit auch zur Therapiebewertung liefern können (Grobe et al. 2008). Besonders vorteilhaft bei der Nutzung von Sekundärdaten ${ }^{1}$ ist, dass die Forschungsfrage keinen Einfluss auf die Daten hat, so dass Ergebnisverzerrungen, die durch Setting, Antwortverhalten oder Nichtteilnahme von Probanden verursacht werden könnten, wegfallen. Der wissenschaftliche Wert der Daten von Krankenkassen resultiert maßgeblich auch daraus, dass sich personenbezogene sozialstatistische Daten mit Informationen aus unterschiedlichen Leistungssektoren verknüpfen lassen (Grobe \& Ihle 2005). Dazu kommt, dass der Umfang der Datensätze großer gesetzlicher Krankenkassen auch die Analyse seltener Ereignisse ermöglicht (Geyer 2003).

Grundsätzlich regeln die Vorschriften in $\mathbb{} 303 f \mathrm{fGB} V$ in Verbindung mit $\mathbb{} 75$
SGB X, wann und von wem Krankenkassenroutinedaten für wissenschaftliche Forschungszwecke genutzt werden dürfen. Eine Nutzung der Daten ist nur dann zulässig, wenn die betroffenen Versicherten zustimmen oder aber die Daten vollständig anonymisiert vorliegen. Die Übermittelung der Daten bedarf ferner der vorherigen Genehmigung durch die oberste Bundes- oder Landesbehörde, die für die jeweilige Krankenkasse als Aufsicht zuständig ist. Hierbei müssen Forschungsvorhaben, Datenumfang und Speicherungsdauer genau bezeichnet werden und dem Zweck des Forschungsvorhabens entsprechen. Großer Vorteil ist die grundsätzliche Verfügbarkeit über mehrere Jahre, wobei beschränkend wirkt, dass Daten nur für eine bestimmte Zeit gespeichert werden. Die Löschungsfristen richten sich nach dem Leistungsbereich und sind im $\$ 304$ SGB $\mathrm{V}$ gesetzlich geregelt. Hinzu kommen Einschränkungen, die durch das freie Krankenkassenwahlrecht innerhalb der gesetzlichen Krankenkassen $(\mathbb{\$} 173-175$ SGB V) bedingt sind: so ist es nur unter sehr erschwerten Bedingungen möglich, die kompletten Daten von Versicherten $\mathrm{zu}$ verfolgen, die während einer betrachteten Periode bei unterschiedlichen Krankenkassen versichert waren.

Geyer (2003) führt aus, dass bei der Analyse von Krankenkassendaten eine Reihe von Punkten zu beachten sind, um fehlerhafte Analysen und die Veröffentlichung von Artefakten zu vermeiden. So genügten die Dateninhalte oft nicht den tatsächlichen Bedürfnissen der Forscher, da sich fehlende Details oder unklare Erfassungskategorien nicht nachträglich „heilen“ lassen. Unzureichende Dokumentationsstandards und daraus resultierend die Unmöglichkeit, den Prozess der Datenbeschaffung sowie die Datenqualität und Validität zu beurteilen, können Forschungsergebnisse unzuverlässig machen. Auch nicht identifizierte Über- oder Unterkodierungsanreize sowie Änderungen in der Verschlüsselungssystematik können leicht zu falschen Schlussfolgerungen führen. Wenn die Daten in einem Zustand sind, der arbeitsintensives Aufarbeiten not-

1 Als Sekundärdaten werden solche Daten bezeichnet, die ursprünglich für andere Zwecke erhoben und gespeichert wurden. 
Abbildung 1: Teilprozesse des Hypertoniemanagements

\begin{tabular}{|c|c|c|c|}
\hline 1. Erkennung & 2. Diagnos tik & 3. Therapie & 4. Verlaufskontrolle \\
\hline $\begin{array}{l}\text { Wird eine Hypertonie } \\
\text { erkannt? }\end{array}$ & $\begin{array}{l}\text { Werden Befunde } \\
\text { korrekt abgeklärt? } \\
\text { Wird das Gesamtrisiko } \\
\text { ermittelt? }\end{array}$ & $\begin{array}{l}\text { Wird eine } \\
\text { leitlinienadäquat und } \\
\text { wirtschaftliche } \\
\text { Therapie eingeleitet? }\end{array}$ & $\begin{array}{l}\text { Wird der Erfolg der } \\
\text { Therapie regelmäßig } \\
\text { überprüft und die } \\
\text { Therapie ggf. } \\
\text { angepasst? Treten } \\
\text { Folgeschäden auf? }\end{array}$ \\
\hline
\end{tabular}

Quelle: Eigene Abbildung auf Basis der Leitlinien der Deutschen Gesellschaft für Hypertonie und Prävention - Deutsche Hochdruckliga e.V. (DHL) und der Arzneimittelkommission der deutschen Ärzteschaft (Akd̈̈)

wendig macht, kann ein erhoffter Zeitvorteil schnell wieder aufgehoben sein.

Aufgrund der beschriebenen Vor- und Nachteile wurden zu Beginn des neuen Jahrtausends in den USA und auch in Europa Richtlinien über die Nutzung von Sekundärdaten entwickelt ${ }^{2}$. Dies belegt sowohl den Stellenwert von Routinedatenauswertungen als auch die Notwendigkeit der korrekten Anwendung wissenschaftlich fundierter Methoden bei der Auswertung dieser Daten.

\section{Gegenüberstellung von Informationen in Routinedaten mit Aspekten einer leitliniengerechten Hypertonie-Therapie}

Zentrales Ziel der Hypertonietherapie ist es, durch eine Einstellung des Blutdrucks die Morbidität und Mortalität aufgrund möglicher Folgeerkrankungen wie z.B. Herzinfarkt, Schlaganfall oder Niereninsuffizienz zu senken. Die Blut- drucksenkung ist dabei als Teil einer Gesamttherapie anzusehen, die parallel alle weiteren ggf. vorliegenden kardiovaskulären Risikofaktoren einbeziehen muss. Um entscheiden zu können, ob und in welchem Umfang Empfehlungen aus Leitlinien gefolgt wird, sind folglich Informationen nötig, die neben den möglichen Therapieschritten auch die grundlegenden Entscheidungskriterien widerspiegeln. Hierzu wurde der Versorgungsprozess zunächst in die Teilprozesse Erkennung, Diagnostik, Therapie und Verlaufskontrolle der Hypertonie eingeteilt (Abbildung 10).

Im Anschluss wurden die beiden Leitlinien der DHL und der AKd Ä hinsichtlich ihrer diagnostischen und therapeutischen Empfehlungen verglichen. Dabei zeigt sich, dass die Leitlinien geringfügige Differenzen aufweisen, was beispielsweise Reihenfolge und Zeitpunkt medikamentöser Therapieempfehlungen oder auch die Höhe des Ziel- blutdruckes betrifft. Dies ist teilweise auf unterschiedliche Erscheinungszeitpunkte der Leitlinien, häufiger aber auf eine unterschiedliche Berücksichtigung und Bewertung vorliegender Evidenz aus Studien zurückzuführen. Insgesamt empfiehlt die HDL eher eine frühere und stärkere Blutdrucksenkung als die AkdÄ, auch werden die verfügbaren Antihypertensiva etwas anders bewertet. Im Kern ihrer Empfehlungen stimmen die Leitlinien jedoch überein und die Unterschiede in Details spielen für die hier gestellte Frage der Abbildbarkeit in Routinedaten zunächst keine Rolle. Bei einer Bewertung

2 Die American Medical Informatics Society konzipierte Richtlinien für die Nutzung gesundheitsbezogener Sekundärdaten. In Deutschland erarbeitete und veröffentlichte die Arbeitsgruppe Erhebung und Nutzung von Sekundärdaten (AGENS) der Deutschen Gesellschaft für Sozialmedizin und Prävention (DGSMP) die Leitlinie "Gute Praxis Sekundärdatenanalyse (GPS)“, die seit 2012 in der 3. revidierten Fassung vorliegt.

Tabelle 1: Bewertung der Verfügbarkeit von Informationen in GKV-Routinedaten zu Aspekten einer leitlinienadäquaten Erkennung von Hypertoniepatienten

\begin{tabular}{|c|c|c|c|}
\hline Aspekt & $*$ & Anmerkung Chancen & Anmerkung Limitationen \\
\hline $\begin{array}{l}\text { Anteil der Hypertoniker, die von } \\
\text { ihrer Hypertonie wissen }\end{array}$ & -- & & $\begin{array}{l}\text { Wer trotz Wissen nicht zum Arzt geht, erhält auch } \\
\text { keine Diagnose; umgekehrt kann nicht zweifelsfrei } \\
\text { davon ausgegangen werden, daß jeder Patient die } \\
\text { ärztl. Diagnose kennt/akzeptiert. }\end{array}$ \\
\hline $\begin{array}{l}\text { Anteil der Hypertoniker in } \\
\text { ärztlicher Behandlung }\end{array}$ & + & $\begin{array}{l}\text { Die Anzahl der vom Arzt diagnosti- } \\
\text { zierten Hypertoniker lässt sich } \\
\text { anhand der Diagnosekodierung } \\
\text { (ICD I10) bestimmen. Das Ergebnis } \\
\text { kann mit einem Erwartungswert } \\
\text { verglichen werden, wenn die Gruppe } \\
\text { groß genug ist. }\end{array}$ & $\begin{array}{l}\text { Unklare Validität ambulanter Diagnosekodierungen. } \\
\text { Bezugsbasis des Erwartungswertes muss vergleichbar } \\
\text { sein (Altersaufbau, sozialer Status, Geschlecht etc). } \\
\text { Ambulante Diagnosen liegen erst mit einer Zeitverzö- } \\
\text { gerung von ca. } 9 \text { Monaten und maximal rückwirkend } \\
\text { bis zum Jahr } 2004 \text { vor. }\end{array}$ \\
\hline $\begin{array}{l}\text { Regelhafte Blutdruckmessung bei } \\
\text { Praxisbesuch eines Risikopatienten }\end{array}$ & -- & & Leistung in EBM-Versichertenpauschale enthalten \\
\hline
\end{tabular}

* Bewertung der Verfügbarkeit von Informationen zu diesem Aspekt in GKV-Routinedaten: ++ sehr detailliert; + ja; O bedingt bzw. eingeschränkt; -- nein

Quelle: Eigene Darstellung 


\begin{tabular}{|c|c|c|c|}
\hline Aspekte bei der Diagnostik & $*$ & Anmerkung Chancen & Anmerkung Limitationen \\
\hline $\begin{array}{l}\text { Verifikation eines dauerhaft erhöhten } \\
\text { Blutdrucks? }\end{array}$ & -- / O & $\begin{array}{l}\text { Kennzeichnung als Verdachts- bzw. } \\
\text { gesicherte Diagnose nach ICD-10 }\end{array}$ & $\begin{array}{l}\text { Keine Angaben darüber, worauf die Diagnose } \\
\text { fußt ( } 24 \text { Std. Messung oä) }\end{array}$ \\
\hline $\begin{array}{l}\text { Welcher Schweregrad der Hypertonie } \\
\text { liegt vor? }\end{array}$ & -- & & $\begin{array}{l}\text { Schweregrad der Hypertonie wird nicht } \\
\text { verschlüsselt }\end{array}$ \\
\hline Anamnese & -- & & Grds. keine Angaben in Routinedaten \\
\hline $\begin{array}{l}\text { Wurden weitere Risikoparameter } \\
\text { erhoben? Ggf. Diagnostik? }\end{array}$ & $\mathrm{O}$ & Ggf. EBM- und Laborziffern & $\begin{array}{l}\text { Informationen aus der EBM-Abrechnung } \\
\text { lassen nur sehr begrenzt Aussagen zu }\end{array}$ \\
\hline Ergebnisse der Diagnostik & -- & $\begin{array}{l}\text { Ggf. über parallel verschl. } \\
\text { ICD-Diagnose }\end{array}$ & $\begin{array}{l}\text { Routinedaten enthalten keine direkten Infor- } \\
\text { mationen zu Ergebnissen diagnostischer Tests }\end{array}$ \\
\hline $\begin{array}{l}\text { Wurden manifeste Komorbiditäten, } \\
\text { Endorganschäden ermittelt oder sind } \\
\text { bekannt? }\end{array}$ & $\mathrm{O} /+$ & $\begin{array}{l}\text { Ggf. über parallel verschlüsselte } \\
\text { ICD-Diagnosen oder Arzneimittel- } \\
\text { verordnungen }\end{array}$ & $\begin{array}{l}\text { Ambulant keine Kennzeichnung von } \\
\text { Haupt- und Nebendiagnosen, abhängig von } \\
\text { ICD-Systematik und Kodierqualität }\end{array}$ \\
\hline Patientenpräferenzen & -- & & Keine Angaben in Routinedaten \\
\hline
\end{tabular}

* Bewertung der Verfügbarkeit: ++ sehr detailliert; + ja; O bedingt bzw. eingeschränkt; -- nein

Quelle: Eigene Darstellung

von Ergebnissen wäre allerdings die Frage zu stellen, welche Leitlinie man als Messlatte zugrunde legen will.

Die folgenden vier Tabellen bilden die verschiedene Aspekte einer Hypertoniebehandlung zu jedem Teilprozess (Erkennung, Diagnostik, Therapie und Verlaufskontrolle) ab. Es wird beurteilt, inwiefern Routinedaten der gesetzlichen Krankenkassen Informationen zu dem jeweiligen Aspekt enthalten.

Großer Vorteil der Routinedaten ist, dass sich mit Hilfe des ICD-10-GM Diagnoseschlüssels alle vom Arzt diagnostizierten Hypertoniker identifizieren lassen. Sofern epidemiologische Kenntnisse über eine hinsichtlich Alter und Geschlecht vergleichbare Bevölkerungsgruppe verfügbar sind, lässt sich bestimmen, ob der Anteil der Versicherten mit Hypertoniediagnose dem erwartbaren Anteil von Hypertonikern in der Grundgesamtheit entspricht. Damit ist es möglich, die Behandlungsprävalenz einzuschätzen. Anhand von aktuellen Diagnosedaten der AOK Niedersachsen lässt sich beispielsweise die Behauptung, nur ein sehr geringer Teil der Patienten mit Bluthochdruck würden erkannt und behandelt, nicht aufrecht erhalten (eigene Auswertungen). Die Daten zeigen, dass gerade in den höheren Altersgruppen der Anteil an Versicherten mit Hypertoniediagnose der erwartbaren Prävalenz in der jeweiligen Alters- und Geschlechtsgruppe entspricht.

Nachteilig ist allerdings die unklare Qualität ärztlicher Diagnosekodierun- gen. Zwar sind die Krankenkassen zu Auffälligkeitsprüfungen nach \$296 SGB $\mathrm{V}$ verpflichtet, dies kann aber naturgemäß nicht die Frage umfassen, auf welcher Basis und mit welcher Genauigkeit eine Diagnose kodiert wird. Insbesondere ambulante Diagnosedaten werden als mit einer hohen bzw. unsicheren Fehlerquote belastet angesehen (Giersiepen et al. 2007; Gerste \& Gutschmied 2006), wobei sich die Validität durch die Bemühungen um vollständiges und korrektes Kodieren im ambulanten Sektor in den vergangenen Jahren insgesamt verbessert haben dürfte (Gaßner et al. 2010).

\section{Insbesondere ambulante Diagnosedaten werden als mit einer hohen bzw. unsicheren Fehlerquote belastet angesehen.}

Die Diagnoseprävalenz kann nicht der Morbidität gleichgesetzt werden: neben Änderungen im Kodier- und Dokumentationsverhalten der Ärzte können verbesserte diagnostische Möglichkeiten, die Veränderung von Grenzwerten (z.B. wurden die hypertensiven Grenzwerte in den vergangenen Jahrzehnten deutlich herabgesetzt) oder Veränderungen bei der Speicherungsmöglichkeit und Software in Arztpraxen und Krankenkassen eine Rolle spielen. In der Praxis zeigt sich, dass die Zahl aller ambulant ko- dierten Diagnosen seit ihrer erstmaligen Übermittelung an die Krankenkassen im Jahr 2004 kontinuierlich angestiegen ist (Gaßner et. al 2010). Vergleiche mit zurückliegenden Jahren sind folglich mit großer Vorsicht zu interpretieren, es sollte möglichst auf aktuelle Daten zurückgegriffen werden.

Tabelle zwei verdeutlicht, dass GKVRoutinedaten nur bedingt genutzt werden können, um eine leitlinienadäquate Diagnostik zu prüfen oder um diagnostische Ergebnisse als Risikofaktoren zu berücksichtigen. $\mathrm{Ob}$ beispielsweise die Diagnose Hypertonie auf einer einmaligen Messung des Blutdrucks basiert oder anhand einer LangzeitBlutdruckmessung gestellt wurde, kann anhand von Routinedaten nicht nachvollzogen werden. Auch enthalten die Daten weder entscheidende klinische (z.B. Schweregrad, Nierenfunktionswerte) noch anamnestische (z.B. Unverträglichkeiten) oder persönliche Daten (z.B. Körpergewicht, Raucherstatus) ${ }^{3}$. Anders als bei Herzinsuffizienz, bei der der ICD-Katalog eine Angabe des NYHA-Schweregrades ermöglicht,

3 Anderes gilt für Routinedaten in Arztpraxen: Das AQUA Institut hat im Rahmen des Projektes QiSA Ende 2010 Qualitätsindikatoren für die Behandlung von Bluthochdruck-Patienten vorgelegt, die sich auf dafür gesondert zu erfassende Daten in Arztpraxen beziehen. Dazu gehört etwa die Erstellung eines Hypertonieregisters, dem Anteil der Hypertoniker mit Basisdiagnostik, mit erreichter Normotonie oder mit Schulung (Chenot \& Szecsenyi 2010). 
Tab. 3: Bewertung der Verfügbarkeit von Informationen in GKV-Routinedaten zu Aspekten einer leitlinienadäquaten Therapie bei Hypertoniepatienten

\begin{tabular}{|c|c|c|c|}
\hline Aspekt & $*$ & Anmerkung Chancen & Anmerkung Limitationen \\
\hline $\begin{array}{l}\text { Beratung und Motivation hin- } \\
\text { sichtlich nichtmedikamentöser } \\
\text { Maßnahmen }\end{array}$ & -- & & Leistung in EBM-Pauschale enthalten \\
\hline Medikamentöse Therapie & ++ & $\begin{array}{l}\text { Hypertoniepräparate sind verschrei- } \\
\text { bungspflichtig, somit sind ambulante } \\
\text { Verordnungsdaten vollständig verfügbar } \\
\text { (Ausnahme: Selbstzahler), vergleichswei- } \\
\text { se gute Datenqualität } \\
\text { Zur Dosisabschätzung Annäherung über } \\
\text { DDD und Verordnungsdatum möglich }\end{array}$ & $\begin{array}{l}\text { Anlass der Verordnung unbekannt } \\
\text { Kombinationspräparate: beachten, unter welcher } \\
\text { Wirkstoffobergruppe diese im ATC-Code einsor- } \\
\text { tiert sind } \\
\text { Keine Daten über Arzneimittel, die während eines } \\
\text { stationären Aufenthaltes gegeben wurden }\end{array}$ \\
\hline $\begin{array}{l}\text { Berücksichtigung von Risikofak- } \\
\text { toren, Organschäden, Komorbi- } \\
\text { ditäten }\end{array}$ & $\mathrm{O}$ & $\begin{array}{l}\text { Versichertenstammdaten enthalten die } \\
\text { „Risikofaktoren“ Alter und Geschlecht } \\
\text { Komorbiditäten können anhand statio- } \\
\text { närer Diagnosen, ambulanter Diagnosen } \\
\text { und Arzneimitteldaten eingeschätzt } \\
\text { werden }\end{array}$ & $\begin{array}{l}\text { Keine Ergebnisse von Anamnese oder Labor, keine } \\
\text { Informationen zu Lebensstilfaktoren (z.B. Raucher) } \\
\text { Validität der Diagnosekodierung? } \\
\begin{array}{l}\text { Schweregrad einer Erkrankung nur begrenzt nach- } \\
\text { vollziehbar }\end{array}\end{array}$ \\
\hline $\begin{array}{l}\text { Berücksichtigung weiterer Medi- } \\
\text { kation hinsichtlich Auswahl und } \\
\text { Dosierung der Medikamente }\end{array}$ & + & $\begin{array}{l}\text { Gute Daten verschreibungspfl. } \\
\text { Arzneimitteln, auch wenn von } \\
\text { unterschiedlichen Ärzten }\end{array}$ & $\begin{array}{l}\text { Keine Informationen über OTC-Medikation und } \\
\text { Privatrezepte }\end{array}$ \\
\hline $\begin{array}{l}\text { Berücksichtigung von Patien- } \\
\text { tenpräferenzen hinsichtlich der } \\
\text { Medikamentenauswahl }\end{array}$ & -- & & Keine Angaben in Routinedaten \\
\hline $\begin{array}{l}\text { Berücksichtigung ökonomischer } \\
\text { Aspekte bei der Auswahl der } \\
\text { Medikamente }\end{array}$ & + & $\begin{array}{l}\text { Routinedaten enthalten Preis und somit } \\
\text { Kosten pro DDD } \\
\text { Verordnungsquoten von Generika; Ana- } \\
\text { logpräparate; Substanzklassenvergleiche } \\
\text { über ATC-Code sind möglich }\end{array}$ & $\begin{array}{l}\text { Keine individuellen Einschätzungen möglich (es kön- } \\
\text { nen z.B. gute Gründe vorliegen, einem bestimmten } \\
\text { Patienten ein anderes als das primär empfohlene } \\
\text { Medikament zu verordnen }\end{array}$ \\
\hline
\end{tabular}

* Bewertung der Verfügbarkeit: ++ sehr detailliert; + ja; O bedingt bzw. eingeschränkt; - nein

Quelle: Eigene Darstellung

ist dies bei der Diagnose „Hypertonie“ nicht vorgesehen. Dennoch lassen sich bestimmte Endorganschäden und Komorbiditäten nachvollziehen, indem Kodiagnosen oder Arzneimittelverordnungen herangezogen werden. Voraussetzung ist, dass die entsprechende Diagnose nach ICD-10-GM verschlüsselbar ist und auch korrekt verschlüsselt wurde. Bei der Nutzung von Daten aus dem ambulant-ärztlichen Bereich muss bedacht werden, dass diese erst mit erheblichen Zeitverzug bei den Krankenkassen vorliegen. Neben den Diagnosen erhalten die gesetzlichen Krankenkassen von den Kassenärztlichen Vereinigungen Abrechnungsziffern gemäß des Einheitlichen Bewertungsmaßstabes (EBM), in dem Pauschalen oder Einzelleistungen nach der jeweils gültigen Gebührenord- nung verzeichnet sind (siehe $\mathbb{} 295$ SGB V). Bestimmte Abrechnungsziffern im EBM sind nur für bestimmte Arztgruppen abrechnungsfähig und können dort wiederum nur unter bestimmten Bedingungen abgerechnet werden. Der Inhalt und die Abrechnungsfähigkeit einzelner Leistungsziffern ist in den einzelnen Bundesländern zum Teil unterschiedlich geregelt und regelmäßig Gegenstand von Reformen, was die Eignung für wissenschaftliche Fragestellungen sehr einschränkt (Grobe et al. 2010). Zudem wurde die Vergütung niedergelassener Haus- und Fachärzte durch die Reform des EBM im Jahr 2008 stark pauschalisiert. Seitdem werden die im Rahmen der hausärztlichen Versorgung üblicherweise erbrachten Leistungen eines Behandlungsfalles mit einer Versi- chertenpauschale vergütet. Die Höhe der Pauschale hängt vom Alter und Art der Inanspruchnahme des Versicherten $a b$, zudem können ein Chronikerzuschlag sowie bestimmte Einzelleistungen bzw. Komplexe für besonders förderungswürdige Leistungen abgerechnet werden. Die Messung des Blutdrucks ist Teil der Versichertenpauschale und wird nicht einzeln ausgewiesen. Analog sind die im fachärztlichen Bereich „Grundpauschale“ genannten Leistungspauschalen für Fachärzte ebenfalls nur einmal im Behandlungsfall abrechnungsfähig. Die Pauschalierung hat für die Betrachtung von Routinedaten erhebliche Konsequenzen: es kann weder nachvollzogen werden, wie viele Arztkontakte ein Patient innerhalb eines Quartals oder Jahres tatsächlich hatte noch welche Einzel- 
leistungen tatsächlich erbracht wurden (Grobe et al. 2010 S. 40f). Ebensowenig wäre nachvollziehbar, ob diagnostische Ergebnisse aus früheren Untersuchungen bereits vorliegen. Der Umsatz hausärztlich tätiger Allgemeinmediziner setzt sich zu fast 70 Prozent aus Versichertenpauschalen und Zuschlägen für chronisch Kranke zusammen. Die Einführung der Pauschalen erschwert somit insbesondere den Blick in den hausärztlichen Bereich (Wido 2010). Weitere Änderungen könnten langfristig durch die Umsetzung von Selektivverträgen (z.B. Hausarztzentrierte Versorgung nach $\mathbb{S} 73 \mathrm{~b}$ SGB V) zwischen Krankenkassen und Ärzten entstehen. Hierbei ist sowohl eine Verbesserung der Datenbasis bei einzelnen Kassen denkbar, als auch die Verringerung der auf dem üblichen Wege abgerechneten Leistungen mit der Konsequenz, dass Abrechnungswege noch komplexer und unübersichtlicher werden.

Auf individueller Ebene lässt sich aus GKV-Routindedaten weder der Erwartungszeitpunkt eines leitliniengemäßen Beginns einer pharmakologischen Behandlung einschätzen noch die „Leitliniengerechtigkeit" einer verwendeten Dosis beurteilen. Um im Einzelfall direkt beurteilen zu können, ob eine Behandlung leitlinienadäquat vorliegt, wäre es notwendig, dass die Diagnose Hypertonie eine wirkliche Entität, also eine einheitliche Konstante darstellt, die Behandlung also die Variable wäre, die man darüber in ihrer Qualität beurteilen könnte. Dies ist aber nicht der Fall, da es bei Hypertonie eine „Einheitlichkeit“ des Gesundheitsproblems nicht gibt und die Therapieentscheidung entscheidend von Faktoren abhängt, die nicht in den Daten abgebildet sind. Die Tabellen zwei und drei zeigen, dass viele Aspekte, die für eine Bewertung der Bedarfsgerechtigkeit der Medikation eines Einzelfalles benötigt würden, in GKV-Daten nicht vorliegen. Um dennoch die Leitliniengerechtigkeit der Versorgung beurteilen zu können, müssen sich interindividuelle Unterschiede ausgleichen können. Dies wird durch die Betrachtung einer größeren Gruppe möglich, sofern bestimmte (statistische) Bedingungen erfüllt sind. Wie Abholz (2007) ausführt, reicht dazu die Größe einer einzelnen Arztpraxis im Allgemeinen nicht aus. Hierin liegt der große Vorteil von Sekundärdaten: durch die Vollständigkeit und Größe der Datensätze können sich individuelle Unterschiede im statistischen Mittel ausgleichen und eine Gesamtbewertung wird möglich.

Da antihypertensive Medikamente verschreibungspflichtig sind, liegen ins- gesamt gute Daten zur Arzneimitteltherapie vor. Die Informationen umfassen u.a. Wirkstoff, Abgabemenge, Preis und Verordnungsdatum. Mit Ausnahme der Medikamente, die während eines stationären Aufenthalts gegeben werden, ist also grundsätzlich die gesamte antihypertensiv wirksame Medikation pseudonymbezogen verfügbar. Eine systematische Lücke in den Verordnungsdaten könnte nur durch Selbstbehalttarife entstehen, wenn sich der Versicherte entscheidet, eine Verordnung auf einem Privatrezept ausstellen zu lassen, um seine finanziellen Vorteile aus dem Tarif nicht zu gefährden. Diese Tarife sind allerdings sehr unattraktiv für Menschen mit einer chronischen Erkrankung.

Nachteilig ist, dass sich die Verordnungsdaten keiner Diagnose direkt zuordnen lassen. Antihypertensive Medikamente sind häufig für weitere Indikationen des Herz-Kreislauf-Systems zugelassen. Folglich kann nicht unterschieden werden, ob ein Patient ein bestimmtes Mittel speziell für die Behandlung der Hypertonie oder für eine andere Krankheit erhalten hat. Das Endergebnis - der Patient erhält einen Wirkstoff, der antihypertensiv

Tab. 4: Bewertung der Verfügbarkeit von Informationen in GKV-Routinedaten zu Aspekten einer leitlinienadäquaten Verlaufskontrolle bei Hypertoniepatienten

\begin{tabular}{|c|c|c|c|}
\hline Aspekt & $*$ & Anmerkung Chancen & Anmerkung Limitationen \\
\hline $\begin{array}{l}\text { Regelmäßige Kontrolle des Blut- } \\
\text { drucks }\end{array}$ & -10 & $\begin{array}{l}\text { Es kann nur nachvollzogen werden, ob eine } \\
\text { Diagnose in jedem Quartal vorliegt; }\end{array}$ & $\begin{array}{l}\text { nicht aber, ob tatsächlich Blutdruck gemes- } \\
\text { sen wurde und mit welchem Ergebnis }\end{array}$ \\
\hline Regelmäßige Folgeverordnungen & ++ & $\begin{array}{l}\text { Zeitpunkt, Art und Menge der Verordnung } \\
\text { kann in Arzneimitteldaten nachvollzogen } \\
\text { werden }\end{array}$ & \\
\hline $\begin{array}{l}\text { Motivation und Schulung des } \\
\text { Patienten durch den Arzt }\end{array}$ & -- & & Enthalten in EBM-Versichertenpauschale \\
\hline $\begin{array}{l}\text { Compliance des Patienten bei } \\
\text { Lebensstilveränderungen }\end{array}$ & -- & & Nicht verfügbar \\
\hline $\begin{array}{l}\text { Compliance des Patienten bei } \\
\text { Medikamenteneinnahme }\end{array}$ & -10 & $\begin{array}{l}\text { Ggf. über Verordnungsmenge und - } \\
\text { häufigkeit nachvollziehbar; }\end{array}$ & $\begin{array}{l}\text { allerdings bleibt unklar, welche Gründe bei } \\
\text { Abbruch vorliegen (z.B. medizinische Been- } \\
\text { digung der Therapie) }\end{array}$ \\
\hline $\begin{array}{l}\text { Überweisung zum Facharzt bei } \\
\text { therapieresistenter Hypertonie }\end{array}$ & -10 & Facharztinanspruchnahme sichtbar & $\begin{array}{l}\text { Grund für Überweisung an Facharzt ist } \\
\text { unbekannt und muss nicht aufgrund der } \\
\text { Hypertonie erfolgt sein }\end{array}$ \\
\hline $\begin{array}{l}\text { Stationärer Aufenthalt aufgrund } \\
\text { Hypertonie oder hypertoner Krise }\end{array}$ & + & $\begin{array}{l}\text { Kodierrichtlinien für stationäre Diagnosen; } \\
\text { fünfte Stelle im ICD-10-GM ermöglicht } \\
\text { ergänzend die Verschlüsselung einer hyper- } \\
\text { tonen Krise; }\end{array}$ & Unterschiede bei Diagnosedokumentation? \\
\hline
\end{tabular}

* Bewertung der Verfügbarkeit: ++ sehr detailliert; + ja; O bedingt bzw. eingeschränkt; - nein

Quelle: Eigene Darstellung 
wirkt - bleibt davon allerdings unberührt.

\section{Mit Verordnungsdaten kann nicht unterschieden werden, ob ein bestimmtes Mittel für Hypertonie oder für eine andere Krankheit verordnet wurde.}

Dennoch wäre zu überlegen, die $\mathrm{Pa}$ tienten nach Komorbidität zu stratifizieren und die Therapieempfehlungen wesentlicher Komorbiditäten in eine Beurteilung mit einzubeziehen. Während sich die Verteilung der Blutdruckschweregrade innerhalb einer Gruppe über eine statistische Normalverteilung schätzen lässt, können für die Einschätzung von Risikoprofil und Komorbidität eines Einzelnen das Vorliegen weiterer Diagnosen aus verschiedenen Bereichen verwendet werden. GKV-Routinedaten ermöglichen zudem die Anwendung von Komorbiditäts-Indizes, da bei Krankenkassen Informationen aus den unterschiedlichen Bereichen zusammenfließen und sich mit den ebenfalls vorliegenden sozialdemographischen Informationen verknüpfen lassen.

Möglich ist es somit, Verordnungshäufigkeiten und -kombinationen $\mathrm{zu}$ vergleichen: werden beispielsweise primär solche Wirkstoffe verordnet, die als Hauptwirkstoffe gelten? Wie viele und welche Wirkstoffe nimmt ein Patient parallel ein, wie häufig liegen ungünstige Kombinationen vor? Sind Komorbiditäten vorhanden, die möglicherweise eine Kontraindikation für bestimmte Wirkstoffe darstellen? Ein Beispiel: Im Einzelfall kann es medizinisch begründet sein, dass ein Patient ausschließlich ein antihypertensives Reservepräparat erhält oder dass ein hypertensiver Asthmatiker mit einem Betablocker behandelt wird, obwohl die Verordnung eines Betablockers bei Asthma als absolute Kontraindikation gilt. Allerdings sollten solche Fälle bei der Betrachtung einer Gesamtgruppe hinreichend selten vorkommen. Ein Hinweis auf Fehlversorgung läge etwa dann vor, wenn Hypertoniker mit der weiteren Erkrankung Asthma genauso häufig oder öfter mit Betablockern behandelt würden als Hypertoniker ohne
Asthma oder wenn alle Patienten einer Praxis mit Reservepräparaten behandelt würden. Ebenfalls möglich ist die summarische Betrachtung von Indikatoren hinsichtlich einer wirtschaftlichen Verordnungsweise.

Wie Tabelle 4 verdeutlicht, ist auch die Bewertung der hypertensiven Verlaufskontrolle nur bedingt möglich. Es kann nachvollzogen werden, ob jedes Quartal eine Diagnose „Hypertonie“ vorliegt, der Patient also mindestens einmal beim Arzt gewesen ist. Nachvollzogen werden kann auch, ob regelmäßig ein Rezept in der Apotheke eingelöst wurde. Ob der Patient das verordnete Medikament jedoch regelmäßig einnimmt, bleibt offen. Die Therapieadherence wird im Bereich der Hypertonie generell als schlecht eingeschätzt (Vrijens et al. 2008). Indirekt könnte eine fehlende Folgeverordnung darauf hinweisen, dass ein Patient seine Medikamente nicht einnimmt - da aber nicht jede Hypertonie medikamentös behandelt werden muss, bleibt unklar, ob das Fehlen einer Folgeverordnung auf mangelnde Compliance des Patienten zurückgeht oder bewusster Teil der Therapie ist. Nur bedingt kann betrachtet werden, ob eine Überweisung zum Facharzt erfolgte. Dass und welcher Facharzt in einem Quartal aufgesucht wurde, ist in den Daten sichtbar, nicht jedoch die Information, ob eine Überweisung zum Facharzt aufgrund der Hypertonie erfolgte. Sofern man stationäre (Folge-)Aufenthalte in die Betrachtung integrieren will, ist eine Identifikation der Fälle über die Aufnahme- oder Entlassungsdiagnose möglich. Gemäß der Deutschen Kodierrichtlinien 2012 soll etwa unterschieden werden, ob ein Patient mit einer Herzkrankheit aufgenommen wurde, die in kausalem Zusammenhang mit einer Hypertonie steht oder ob die Erkrankungen ohne kausale Beziehung vorliegen. Vorausgesetzt, dass auch die fünfte Stelle im ICD-10-GM ausgefüllt wurde, kann der Anteil an Patienten bestimmt werden, die mit hypertensiver Krise aufgenommen wurden. Beachtet werden sollte, dass die Angabe von Diagnosen primär der Abrechnung der Krankenhäuser mit den Krankenkassen dient. Welche Fallpauschale (DRG)

für einen Patienten gezahlt wird, wird unter anderem auf Basis der vorliegenden Diagnosen bestimmt. Ob und in welchem Jahr eine Diagnose eine DRG auslöst, könnte daher die Kodierungsgenauigkeit beeinflussen.

\section{Fazit}

GKV-Routinedaten erlauben oft eine realistische Beurteilung von Nutzungsstrukturen und Gesundheitsleistungen, da sie gerade in dem Zusammenhang erhoben worden sind, den man untersuchen will: unter Alltagsbedingungen. Die Gegenüberstellung von Aspekten einer leitliniengerechten Diagnostik und Therapie bei Bluthochdruck mit Krankenkassenroutinedaten zeigt jedoch, dass eine pauschale Beantwortung der Frage, ob und welcher Teil der Patienten leitlinienadäquat behandelt wird, mit den in Routinedaten verfügbaren Informationen kaum möglich ist. Weder der Schweregrad der Hypertonie noch entscheidende diagnostische Informa-

Ob und welcher Teil der Patienten leitlinienadäquat behandelt wird, lässt ich mit GKV-Routinedaten nicht pauschal beantworten.

tionen sind in den Daten sichtbar. Ferner kommt nichtmedikamentösen Maßnahmen bei der Hypertonietherapie ein hoher Stellenwert zu, was zugleich eine Validierung der Diagnose mit Arzneimittelverordnungen erschwert. Um annähernd diskutieren zu können, ob den Leitlinien in einem individuellen Fall gefolgt wurde, müssten die Daten um Schweregrad, Risikofaktoren und weitere medizinische Parameter ergänzt werden. Individuell beurteilbar sind mit Hilfe von Routinedaten nur solche Fälle, bei denen die Daten auf eine Therapie außerhalb jedes Korridors hinweisen.

Der Nutzen von Routinedaten sollte dennoch nicht gering geschätzt werden: Während der einzelne Arzt häufig nur über Informationen aus seinem eigenen Behandlungsprozess verfügt, fließen bei Krankenkassen Informationen aus allen Sektoren zusammen. Sie spiegeln relativ verlässlich die Versorgungsrealität für die Versicherten wider, und 
umfassen auch solche Patienten, die aufgrund von Multimorbidität, Immobilität oder Alter in klinischen Studien oder Befragungen unterrepräsentiert sind. Über die dokumentierten ärztlichen Diagnosen und Arzneimittelverordnungen in Kombination mit Alter und Geschlecht lassen sich Komorbiditäten und Risikofaktoren zumindest grob einschätzen und summatorische Behandlungsprozesse darüber beurteilen. Folglich lässt sich die aggregierte bzw. durchschnittliche Behandlung einer Gruppe betrachten, die dann mit einem anhand von Leitlinienempfehlungen gebildeten Erwartungskorridor verglichen werden kann. Dies betrifft insbesondere die in vergleichsweise guter Qualität vorliegenden Daten zur medikamentösen Therapie. Ferner lässt sich der Frage nachgehen, ob sich hinsichtlich soziodemographischer oder regionaler Merkmale, adjustiert nach Komorbidität, Unterschiede in der Versorgung finden. Je eindeutiger eine Diagnose gestellt und kodiert werden kann und je spezifischer die Therapie, desto besser ist eine Beurteilung über Routinedaten möglich.

Insgesamt lässt sich festhalten, dass Routinedaten - bei allen aufgezeigten Begrenzungen - ein wichtiger Baustein in der Versorgungsforschung sind. Derzeit gibt es keine anderen Daten in diesem Umfang und in dieser Breite. Das Erkenntnisdefizit, das hinsichtlich der Umsetzung von Leitlinien existiert, kann mit Hilfe dieser Daten zwar nicht komplett, aber doch für einige Teilsaspekte verkleinert werden. $\mathrm{Ob}$ und wie weit die Nutzung dieser Daten möglich ist, hängt von Verfügbarkeit, Validität und Aussagekraft einzelner Parameter $\mathrm{ab}$ und muss für jede Fragestellung gesondert geprüft werden. Beim Umgang mit GKV-Routinedaten sollten daher die notwendigen Kapazitäten und die spezifische Fachkenntnisse nicht unterschätzt werden, die benötigt werden, um Daten aufzuarbeiten und mögliche „Fallstricke“ bei der Interpretation der Ergebnisse zu erkennen und zu vermeiden.

\section{Literatur}

Abholz HH (2006): Epidemiologische und biostatistische Aspekte der Allgemeinmedizin. In: Kochen MM: Allgemeinmedizin und Familienmedizin. 3. Auflage, Stuttgart: Thieme, S. 507-23.

AkdÄ (Arzneimittelkommission der deutschen Ärzteschaft) (2004): ARTERIELLE HYPERTONIE 2. Auflage, Köln: Arzneimittelkommission der deutschen Ärzteschaft.

Deutsche Hochdruckliga \& Deutsche Gesellschaft für Hypertonie und Prävention (2011): Neue Entwicklungen in der Hochdrucktherapie: Eine Bewertung durch die Deutsche Hochdruckliga e.V. DHL ${ }^{\circledR}$ Deutsche Gesellschaft für Hypertonie und Prävention. Über die Webseite: http://www.hochdruckliga.de/tl files/content/ dhl/downloads/DHL-Leitlinien-2011.pdf

Deutsche Hochdruckliga \& Deutsche Gesellschaft für Hypertonie und Prävention (2008): Leitlinien zur Behandlung der Arteriellen Hypertonie. Stand 1. Juli 2008. Berlin: Hochdruckliga.

Deutsche Hochdruckliga (DHL) (2009): Weiter hin erhebliche Unterversorgung von Bluthochdruckpatienten. Presseunterlagen zur Pressekonferenz am 7. Oktober 2009, Tageszentrum im Hause der Bundespressekonferenz, Berlin.

Fitzgerald SP, Bean NG (2010): An analysis of the interactions between individual comorbidities and their treatments--implications for guidelines and polypharmacy. J Am Med Dir Assoc 7:475-84.

Frank W, Konta B (2005): Bluthochdruckleitlinien und ihre Auswirkungen auf das Gesundheitssystem. HTA Bericht 22. Köln: DAHTA@DIMDI.

Gaßner M, Arndt V, Fischer L, Göpffarth D (2010): Sind die Diagnosezahlen nach Einfüh rung des morbiditätsorientierten Risikostruk turausgleich angestiegen? Gesundheits- und Sozialpolitik 64(6):11-19

Gerste B, Gutschmidt S (2006): Datenqualität von Diagnosedaten aus dem ambulanten Bereich. Gesundheits- und Sozialpolitik 60/34):29-43

Geyer S (2003): Forschungsmethoden in den Gesundheitswissenschaften. Weinheim, München: Juventa.

\section{Giersiepen K, Pohlabeln H, Egidi G, Pigeot I} (2007): Quality of diagnostic ICD coding for outpatients in Germany. Bundesgesundheits bl Gesundheitsforsch Gesundheitsschutz 50:1028-38

Grobe G, Dörning H, Schwartz FW (2008): GEK-Report ambulant-ärztliche Versorgung 2008. St. Augustin: Asgard-Verlag.

Grobe G, Dörning H, Schwartz FW (2010): Barmer GEK Arztreport. St. Augustin: AsgardVerlag

Heller G, Günster C (2008): Mit Routinedaten Qualität in der Medizin sichern. GGW 9(1):26-34
Mancia G, Laurent S, Agabiti-Rosei E, Amb rosioni E, Burnier M, Caulfield MJ, Cifkova R, Clement D, Coca A, Dominiczak A, Erdine S, Fagard R, Farsang C, Grassi G, Haller H, Heagerty An, Kjeldsen SE, Kiowski W, Mallion MJ, Malolis A, Narkiewicz K, Nilsson P, Olsen MH, Rahn, KH, Redon J, Rodicio J, Ruilope L, Schmieder RE, Struijker-Boudier HAJ, Zwieten PA, Viigimaa M, Zanchetti A (2009): Reappraisal of European guidelines on hypertension management: a European Society of Hypertension Task Force document. J Hypertens 27:2121-58.

Meisinger C, Heier M, Volzke H, Löwel H, Mitusch R, Hense H-W, Lüdemann J (2006): Regional disparities of hypertension prevalence and management within Germany. J Hypertens 24:293-99.

Murphy S, Friesner D, Rosenman R (2009): Determining factors in the treatment choice of patients with hypertension with complications and secondary hypertension. IJHCQA 22:322-39.

Neugebauer EAM, Icks A, Schrappe M (2010): Memorandum III: Methoden für die Versorgungsforschung (Teil 2). Gesundheitswesen 72:739-48

RKI (2008): Hypertonie. Gesundheitsberichterstattung des Bundes - Heft 43. Berlin: RKI.

Schubert I, Ihle P, Köster I (2005): Verwendung von GKV-Diagnosen in der Sekundärdatenforschung. In: Swart E, Ihle P (Hrsg.): Routinedaten im Gesundheitswesen. Bern: Hans Huber. S. $235-242$.

Selbmann HW, Mohr VD (2008): “Monitoring von Gesundheit und Gesundheitsversorgung in Deutschland - der Datenpool der externen Qualitätssicherung nach $\S 137$ SGB V". In: Fuchs C, Kurth BM, Scriba PC (Hrsg.): Report Versorgungsforschung. Köln: Deutscher Ärzte-Verlag. S. $143-8$

Vrijens B, Vincze G, Kristanto P, Urquhart J, Burnier M (2008): Adherence to prescriped antihypertensive drug treatments: longitudinal study of electronically compiled dosing histories. BMJ 336:1114-7.

Wehling M (2010): Das Geld im Gesundheitswesen reicht noch lange, wenn die Verschwendung aufhört. Monitor Versorgungsforschung $3(1): 33-7$.

WidO (2010): Erschwerter Einblick in die Versorgung. GGW 10(2):6.

Wittchen HU, Krause P, Höfler M, Pfister H, Ritz E, Göke B, Lehnert H, Tschöpe D, Kirch W, Pittrow D, Sharma AM, Bramlage P, Küpper B, Unger T (2003): Arterielle Hypertonie, Diabetes mellitus und assoziierte Erkrankungen in der Allgemeinarztpraxis. Fortschritte der Medizin 121:(Originalien Sonderheft I/2003)19-27.

ZI (2010): Die 50 häufigsten ICD-10-Schlüsselnummern nach Fachgruppen. Berlin, 2010. Erreicht unter < http://www.zi-berlin.de> 